\title{
Optimal utilization of mechanical circulatory support and transplant resources in the comprehensive treatment of terminal heart failure
}

\author{
L Svetina*, M Petricevic, B Biocina
}

From 23rd World Congress of the World Society of Cardio-Thoracic Surgeons

Split, Croatia. 12-15 September 2013

\section{Background}

Treatment of end-stage heart failure has markedly evolved. With only a limited number of available heart transplants (24 in 2012) in our institution, mechanical circulatory support (MCS) has become an integral part of acute and end-stage heart failure treatment and has improved survival. We report our experience in MCS program since 2008.

\section{Methods}

Short, intermediate and long term MCS can be instituted for different clinical indications, ranging from postcardiotomy circulatory failure, acute cardiogenic or respiratory shock, chronic heart failure in patients not eligible for a transplant to heart transplant function deterioration. From September 2008 to June 2013, 94 patients were eligible for MCS. The majority of MCS patients were adults, 89/94. The adult group of 89 patients underwent 110 MCS procedures, with 16 patients experiencing multiple procedures.

\section{Results}

In the adult group, $66 \%$ of patients were at INTERMACS level 1, critical cardiogenic shock, and 19\% were at level 2, progressive decline. Indications for primary MCS included: acute exacerbation of chronic heart failure, acute cardiogenic shock and respiratory failure. Altogether, procedural success was accomplished in $49.4 \%$ of patients. MCS efficiently bridged 14 patients with heart failure to heart transplantation. In 15 patients, long term support was instituted, either as a destination therapy or as a bridge to heart transplant; 13 received HeartMate II, and 2 patients received HeartWare. One patient with respiratory failure was successfully bridged to lung transplantation. In the pediatric group, $60 \%$ patients were bridged to recovery. Postcardiotomy MCS was used in $33.7 \%$ patients. Patient outcome after 30 days was assessed as: alive (22.4\%), alive on support (13.5\%), dead (64.0\%). The most common perioperative complication was renal failure, in $42.7 \%$ patients.

\section{Conclusion}

MCS has become an integral part of terminal stage heart failure treatment alongside established standard procedures.

Published: 11 September 2013

\section{doi:10.1186/1749-8090-8-S1-P105}

Cite this article as: Svetina et al:: Optimal utilization of mechanical circulatory support and transplant resources in the comprehensive treatment of terminal heart failure. Journal of Cardiothoracic Surgery 2013 8(Suppl 1):P105.

* Correspondence: lucijasv@gmail.com

Department of Cardiac Surgery, University Hospital Centre Zagreb, Zagreb, Croatia

(c) 2013 Svetina et al; licensee BioMed Central Ltd. This is an Open Access article distributed under the terms of the Creative Commons Attribution License (http://creativecommons.org/licenses/by/2.0), which permits unrestricted use, distribution, and reproduction in any medium, provided the original work is properly cited. 